\title{
An Integration Algorithm for Bistatic Radar Weak Target Detection
}

\author{
Jiajun Chang $^{1}$ \\ ${ }^{1}$ School of Electronic Engineering,University of Electronic Science and Technology of China,Chengdu,China
}

\begin{abstract}
The bistatic radar weak target detection problem is considered in this paper. An effective way to detect weak target is the long time integration. However, range migration (RM) will occur due to the high speed. Without knowing the target motion parameters, a long time integration algorithm for bistatic radar is proposed in this paper. Firstly, the algorithm utilizes second-order keystone transform (SKT) to remove range curvature. Then the quadratic phase term is compensated by the estimated acceleration. After that, SKT is used once more and the Doppler ambiguity phase term compensation is performed. At last, the target energy is integrated via FT. Simulations are provided to show the validity of the proposed algorithm in the end.
\end{abstract}

\section{INTRODUCTION}

Bistatic radars have most advantages due to the geometrical properties of the system. Apart from its relative immunity to some forms of attack, bistatic radars can utilize the echo signal, which does not travel over the same path as the transmitted signal, to detect weak targets [1-3].Therefore, this paper discuss the weak target detection methods for bistatic radar.

It is known that the long time integration can increase the signal-to-noise-ratio (SNR) and thus improve the radar detection ability [4-7]. It includes coherent integration and non-coherent integration. By compensating the phase fluctuation between different pulses, the performance of coherent integration is much better than the non-coherent integration. MTD is known as the classic coherent integration method to increase SNR of echo signals. However, for the high speed targets, $\mathrm{RM}$ will occur within the coherent integration interval. Keystone transform (KT) is studied to remove the RM in [8]. Paper [9] proposes an algorithm to integrate the echo signal energy, which is named RFT. However, KT and
RFT can only detect the targets with a constant radial velocity. In order to address targets with radial acceleration, SKT is used to remove the range curvature in paper [10]. However, paper [10] hasn't considered the Doppler frequency ambiguity (DFA) problem, which is bring about by the acceleration.

This paper proposes a long time integration method for bistatic radars, which can solve the RM and DFA problem. Firstly, the algorithm utilizes SKT to remove the range curvature. Then the quadratic phase term is compensated by the estimated acceleration. After that, SKT is used once more and the Doppler ambiguity phase term compensation is performed. Finally, the target energy is integrated via FT.

The remainder of this paper is organized as follows. In Section II, the echo signal model is analyzed. In section III, the integration algorithm is proposed. Simulations are provided to demonstrate the effectiveness of the proposed method in Section IV. Finally, the conclusions are made in Section V. 


\section{ECHO SIGNAL MODEL}

\subsection{Target Motion Model}

The bistatic radar geometry is shown in Figure 1. The bistatic radar is located at the $\mathrm{X}$ coordinate. $(-L, 0,0)$ denotes the location of the transmitter $T_{x}$ and ( $L, 0,0)$ denotes the location of the receiver $R_{x}$. The origin of the coordinates system is located at the middle point of the two radars. The original location of the target is $\left(x_{0}, y_{0}, z_{0}\right) \cdot \vec{V}$ denotes the speed vector of the target.

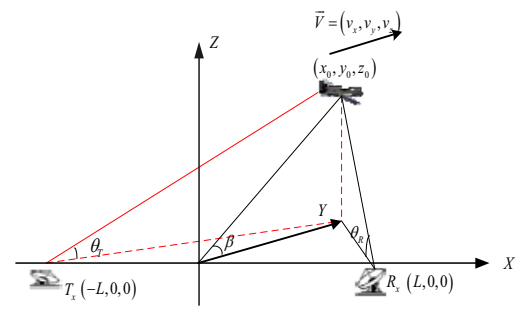

Fig.1. bistatic radar geometry

Suppose that $v_{x}, v_{y}$ and $v_{z}$ denote the coordinate values of target velocity along $\mathrm{X}$ coordinate direction, $\mathrm{Y}$ coordinate direction and $\mathrm{Z}$ coordinate direction, respectively. According to Figure 1, the sum of transmitter-object and object-receiver instantaneous distances can be calculated as follows

$$
\begin{aligned}
R_{s}(t) & =\sqrt{\left(x_{0}+v_{x} t+L\right)^{2}+\left(y_{0}+v_{y} t\right)^{2}+\left(h_{0}+v_{z} t\right)^{2}} \\
& +\sqrt{\left(x_{0}+v_{x} t-L\right)^{2}+\left(y_{0}+v_{y} t\right)^{2}+\left(h_{0}+v_{z} t\right)^{2}}
\end{aligned}
$$

According to the Taylor series theory, neglecting the high order components, we have

$$
R_{s}(t) \approx R_{0}+v t+\frac{1}{2} a t^{2}
$$

Where $R_{0}$ is the sum of transmitter-object and object-receiver initial distances, $v, a$ denote the target velocity and acceleration respectively, which are relative to the direction of bistatic radar line of sight.

\subsection{Echo Signal Model}

Suppose that the radar transmits the linear frequency modulation (LFM) signal as follows

$$
a(\tau)=r \operatorname{ect}\left(\frac{\tau}{T_{p}}\right) \exp \left(j \pi k \tau^{2}\right)
$$

Where $T_{p}$ is the pulse width, $B$ is the band width, $k=B / T_{p}$ denotes the modulation rate.

The echo signal from a point target can be written as follows [11]

$$
\begin{aligned}
& s_{r}\left(\tau, t_{m}\right)=A_{0} \cdot \operatorname{rec} t\left(\frac{\tau-R_{s}\left(t_{m}\right)}{T_{p}}\right) \\
& \times \exp \left(j \pi k\left(\tau-\frac{R_{s}\left(t_{m}\right)}{c}\right)^{2}\right) \times \exp \left(j 2 \pi f_{c}\left(t-\frac{R_{s}\left(t_{m}\right)}{c}\right)\right)
\end{aligned}
$$

Where $A_{0}$ is the echo amplitude, $t_{m}=m T_{r}$ is the slow time, $m=0,1, \cdots, M, M$ is the number of radar pulse, $T_{r}$ is the pulse repetition interval, $c$ denotes the speed of light, $f_{c}$ is the carrier frequency, $R_{s}\left(t_{m}\right)$ can be given by (2).

After demodulation and range compression, the compressed signal in the range frequency-slow time $\left(f_{\tau}-t_{m}\right)$ domain can be given as

$$
\begin{aligned}
& S_{r}\left(f_{\tau}, t_{m}\right)=A_{1} \operatorname{rect}\left(\frac{f_{\tau}}{B}\right) \exp \left[-j \frac{2 \pi\left(f_{\tau}+f_{c}\right)}{c} R_{s}\left(t_{m}\right)\right] \\
&=A_{1} r e c t\left(\frac{f_{\tau}}{B}\right) \exp \left[-j \frac{2 \pi\left(f_{\tau}+f_{c}\right)}{c} R_{0}\right] \times \\
& \exp \left[-j \frac{2 \pi\left(f_{\tau}+f_{c}\right)}{c} v t_{m}\right] \times \exp \left[-j \frac{\pi\left(f_{\tau}+f_{c}\right)}{c} a t_{m}{ }^{2}\right]
\end{aligned}
$$

Where $A_{1}$ is the compressed signal amplitude, the range frequency is $f_{\tau}, \lambda$ denotes wavelength.

Due to the high speed of the target and the low radar pulse repetition frequency (PRF), under-sampling would occur. Therefore, the target speed can be expressed as

$$
v=v_{0}+d \cdot v_{a m b}
$$

Where $v_{a m b}=\lambda / T_{r}$ is the bistatic radar blind velocity, $d$ is the Doppler center ambiguity number, $v_{0}=\bmod \left(v, v_{\text {amb }}\right)$ is the unambiguous velocity which satisfies

$$
\left|v_{0}\right|<\frac{v_{a m b}}{2} \text {. }
$$

Substituting (6) into (5),we have

$$
\begin{aligned}
& S_{r}\left(f_{r}, t_{m}\right)=A_{1} \operatorname{rect}\left(\frac{f_{\tau}}{B}\right) \exp \left[-j \frac{2 \pi\left(f_{\tau}+f_{c}\right)}{c} R_{0}\right] \times \\
& \exp \left[-j \frac{2 \pi\left(f_{z}+f_{c}\right)}{c} V_{0} t_{m}\right] \exp \left[-j \frac{\pi\left(f_{\tau}+f_{c}\right)}{c} a t_{m}{ }^{2}\right] \\
& \times \zeta\left(f_{r}, t_{m}\right)
\end{aligned}
$$

Where $\zeta\left(f_{\tau}, t_{m}\right)=\exp \left[-j \frac{2 \pi\left(f_{\tau}+f_{c}\right)}{c} d v_{a m b} t_{m}\right]=\exp \left[-j \frac{2 \pi f_{\tau}}{c} d v_{a m b} t_{m}\right]$.

Performing the inverse Fourier transform (IFT) of (7) in the range frequency domain, we have

$$
s_{r}\left(\tau, t_{m}\right)=A_{1} B \sin c\left[B\left(\tau-\frac{R_{s}\left(t_{m}\right)}{c}\right)\right] \exp \left[\frac{-j 2 \pi f_{c} R_{s}\left(t_{m}\right)}{c}\right]
$$

(8) shows that the compressed signal envelope moves along $t_{m}$, which is called RM, and the acceleration would result in the DFS. Both RM and DFS would result in 
target energy being defocused.

\section{THE INTEGRATION ALGORITHM}

\subsection{Performing SKT to Remove Range Curvature}

We don't introduce the SKT in detail because it has been discussed in papers [10] before. The formula is

$$
t_{m}=\left(\frac{f_{c}}{f_{c}+f_{\tau}}\right)^{\frac{1}{2}} t_{\alpha}
$$

Where $t_{\alpha}$ is the new slow time after SKT.

Substituting (9) into (7), we have

$$
\begin{aligned}
& S_{r}\left(f_{\tau}, t_{\alpha}\right)=A_{1} \text { rect }\left(\frac{f_{\tau}}{B}\right) \exp \left[-j \frac{2 \pi\left(f_{\tau}+f_{c}\right)}{c} R_{0}\right] \times \\
& \exp \left[-j \frac{2 \pi\left(f_{\tau}+f_{c}\right)}{c} v_{0}\left(\frac{f_{c}}{f_{c}+f_{\tau}}\right)^{\frac{1}{2}} t_{\alpha}\right] \times \exp \left[-j \frac{\pi f_{c}}{c} a t_{\alpha}^{2}\right] \\
& \times \zeta\left(f_{r}, t_{\alpha}\right)
\end{aligned}
$$

Where $\zeta\left(f_{\tau}, t_{\alpha}\right)=\exp \left[-j \frac{2 \pi f_{\tau}}{c} d v_{a m b}\left(\frac{f_{c}}{f_{c}+f_{\tau}}\right)^{\frac{1}{2}} t_{\alpha}\right]$

In (10), it can be seen that the range curvature is removed. However, the RM still exists and the second-order phase term have to be compensated.

\subsection{Compensating the Quadratic Phase Term}

The quadratic phase term should be compensated before correcting the residual RM. In (10), we can see that the phase in the cross range dimension is a chirp signal. Therefore, the method in paper [12] can be used to estimate the acceleration $a$.With the estimated value $\tilde{a}$, we can construct the phase compensation function

$$
H(\tilde{a})=\exp \left[j \frac{\pi f_{c}}{c} \tilde{a} t_{\alpha}^{2}\right]
$$

Multiplying (11) with (10), if $\tilde{a} \approx a$, we have

$$
\begin{aligned}
& S_{r}\left(f_{s}, t_{a}\right) \approx A_{r} \operatorname{rect}\left(\frac{f_{t}}{B}\right) \exp \left[j \frac{2 \pi\left(f_{\tau}+f_{c}\right)}{c} R_{0}\right] \times \\
& \exp \left[-j \frac{2 \pi\left(f_{\tau}+f_{c}\right)}{c} v_{0}\left(\frac{f_{c}}{f_{c}+f_{t}}\right)^{\frac{1}{2}} t_{a}\right] \times \zeta\left(f_{t}, t_{a}\right)
\end{aligned}
$$

\subsection{Correcting the Residual RM}

In (12), the residual RM still exists. The SKT is used to correct the RM once more. Substituting the formula $t_{\alpha}=\left(\frac{f_{c}}{f_{c}+f_{\tau}}\right)^{\frac{1}{2}} t_{\beta}$ into (12), we get

$$
\begin{aligned}
& S_{r}\left(f_{r}, t_{\beta}\right)=\operatorname{Arect}\left(\frac{f_{\tau}}{B}\right) \exp \left[-j \frac{2 \pi\left(f_{\tau}+f_{c}\right)}{c} R_{v}\right] \times \\
& \exp \left[-j \frac{2 \pi f_{c_{c}}}{c} v_{0} t_{\beta}\right] \times \zeta\left(f_{\tau}, t_{\beta}\right)
\end{aligned}
$$

Where $\zeta\left(f_{\tau}, t_{\beta}\right)=\exp \left[-j \frac{2 \pi}{\lambda} d v_{a m b} \frac{f_{\tau}}{f_{c}+f_{\tau}} t_{\beta}\right]$.

\subsection{Compensating the Doppler ambiguity phase term}

In (13), we can see that the range curvature and RM are removed. However, the Doppler ambiguity phase term still exists, which have to be compensated. We construct the compensation function

$$
\xi\left(f_{\tau}, t_{\beta}, d^{\prime}\right)=\exp \left[j \frac{2 \pi}{\lambda} d^{\prime} v_{a n b} \frac{f_{\tau}}{f_{c}+f_{\tau}} t_{\beta}\right]
$$

Multiplying (14) with (13), we have

$$
\begin{aligned}
& S_{r}\left(f_{v}, t_{B}\right)=\operatorname{Arect}\left(\frac{f_{t}}{B}\right) \exp \left[-j \frac{2 \pi\left(f_{\tau}+f_{c}\right)}{c} R_{0}\right] \times \\
& \exp \left[-j \frac{2 \pi f_{c}}{c} v_{v_{\beta}}\right] \times \exp \left[j \frac{2 \pi}{\lambda}(d-d) v_{\operatorname{mab}_{0}} \frac{f_{\tau}}{f_{c}+f_{\tau}} t_{\beta}\right]
\end{aligned}
$$

If $d^{\prime}=d,(15)$ can be expressed as

$$
\begin{aligned}
& S_{r_{r}}\left(f_{\tau}, t_{\beta}\right)=A_{1} \operatorname{rect}\left(\frac{f_{\tau}}{B}\right) \times \exp \left[-j \frac{2 \pi f_{c}}{c} v_{0} t_{\beta}\right] \\
& \quad \times \exp \left[-j \frac{2 \pi\left(f_{\tau}+f_{c}\right)}{c} R_{0}\right]
\end{aligned}
$$

Performing the IFT of (16) in the range frequency $f_{\tau}$ domain, we have

$$
s_{r}\left(\tau, t_{\beta}\right)=A_{2} \sin c\left[B\left(\tau-R_{0} / c\right)\right] \times \exp \left[-j \frac{2 \pi f_{c}}{c}\left(R_{0}+v_{0} t_{\beta}\right)\right]
$$

In (17), it is shown that the signal is a complex sinusoid signal overt $t_{\beta}$. Therefore, the Doppler center ambiguity number can be calculated as follows

$$
\tilde{d}=\underset{d}{\operatorname{argmax}}\left|\underset{t_{\beta}}{\operatorname{Fin}}\left\{\operatorname{IFT}_{f_{\tau}}\left[S_{r}\left(f_{\tau}, t_{\beta}\right) \xi\left(f_{\tau}, t_{\beta}, d^{\prime}\right)\right]\right\}\right|
$$

Where ${ }_{f_{f_{\tau}}}^{I F T}$ denotes the IFT over $f_{\tau}$ dimension, ${ }_{t_{\beta}} T$ denotes the FT over $t_{\beta}$.

With the estimated Doppler center ambiguity number $\widetilde{d}$, the compensation function can be defined as $\xi\left(f_{i}, t_{\beta}, d\right)=\exp \left[j \frac{2 \pi}{\lambda} \omega_{a b b} \frac{f_{\tau}}{f_{c}+f_{\tau}} t_{\beta}\right]$ to compensate the Doppler ambiguity phase term.

\subsection{Integration by FT}

In (17), we know that the RM and DFA are addressed, which is helpful to the integration of the echo signals energy. Applying FT to (17) overt $t_{\beta}$, we have 


$$
s_{r^{\prime}}\left(\tau, t_{\beta}\right)=A_{3} \sin c\left[B\left(\tau-R_{0} / c\right)\right] \times \sin c\left[T\left(f_{t_{\beta}}+\frac{v_{0}}{\lambda}\right)\right]
$$

Where $T=N T_{r}$ is the integration time.

From (19), we know that the target energy is integrated. If the peak of (19) is larger than a

TABLE I. SIMULATION PARAMETERS

\begin{tabular}{|l|l|}
\hline Pulsewidth $T_{p} \quad 10 \mu \mathrm{S}$ & $\begin{array}{l}\text { Transmitter location } \\
(-8000,0,0)\end{array}$ \\
\hline Bandwidth $B \quad 15 \mathrm{MHz}$ & $\begin{array}{l}\text { Receiver location } \\
(8000,0,0)\end{array}$ \\
\hline $\begin{array}{l}\text { Carrier frequency } f_{c} \\
10 \mathrm{GHz}\end{array}$ & $\begin{array}{l}\text { Target initial location } \\
(4000,6000,10000)\end{array}$ \\
\hline $\begin{array}{l}\text { Pulse repetition frequency } \\
f_{r} \quad 300 \mathrm{~Hz}\end{array}$ & $\begin{array}{l}\text { Target initial velocity } \\
(400,600,70)\end{array}$ \\
\hline $\begin{array}{l}\text { Number of integrated pulses } \\
M \quad 150\end{array}$ & SNR -8dB \\
\hline Sampling rate $f_{s} \quad 30 \mathrm{Mt}$ \\
\end{tabular}

given threshold, the target will be detected.

\section{SIMULATIONS}

Simulations are carried out to evaluate the validity of the proposed method. The parameters used in the simulations are listed in Table I. Suppose there is a point target located in the scene.

Figure 2 shows the absolute error of the approximate formula (2). We see that the error is much less than the range sampling cell $\Delta r=\frac{c}{f_{s}}=10$.Therefore, the approximate formula (2) is proper. Figure 3(a) shows the echo after demodulation. The result after range compression is shown in figure 3(b). In figure 3(b), serious range migration occurs due to the target's high speed. Figure (4) shows the result of the proposed method. Figure 4 (a) shows the 2-D image of the result after compensating the Doppler ambiguity phase term, which shows that the RM is removed. Figure $4(\mathrm{~b})$ is the result of the proposed method, which shows the target energy is integrated. The target can be detected if the peak of figure $4(\mathrm{~b})$ is larger than a given threshold. Therefore, the simulation result demonstrates the effectiveness of the proposed algorithm. Figure 5 shows the result of the other methods. Figure 5(a) and Figure 5(b) show that the two methods, i.e. MTD and KT, are invalid because the target radial velocity is changing during the integration interval. Figure 5(c) shows the result of the algorithm proposed in [10]. Due to DFA, the target energy hasn't been integrated in figure 5(c).

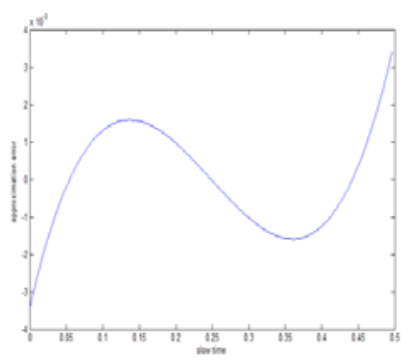

Fig.2 the absolute error of the approximate formula (2)

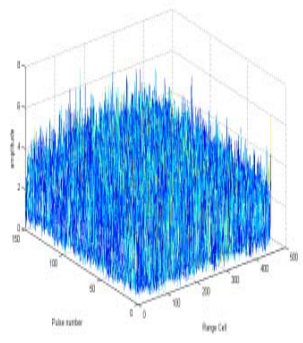

(a)

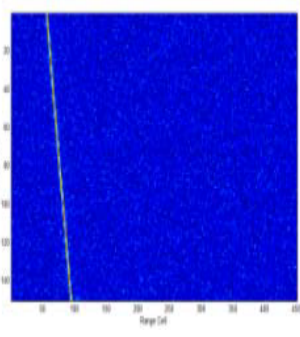

(b)
Fig.3 target echo:(a) The echo after demodulation, (b) The result after range compression

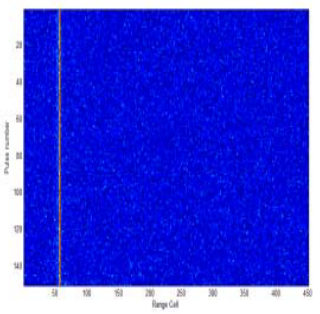

(a)

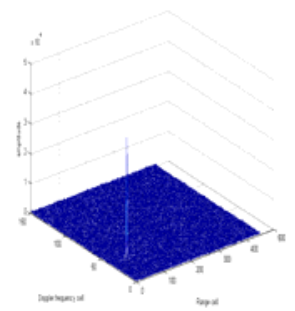

(b)
Fig.4.the result after processing:(a) The 2-D image of the result after compensating the Doppler ambiguity phase term, (b) The result of the proposed method

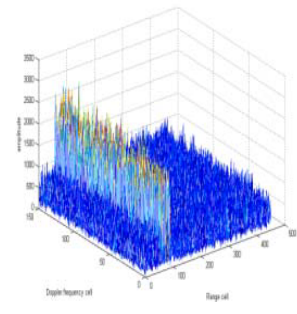

(a)

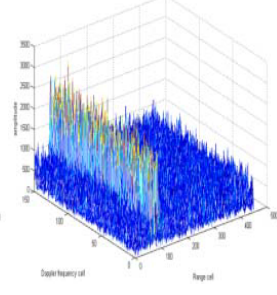

(b)

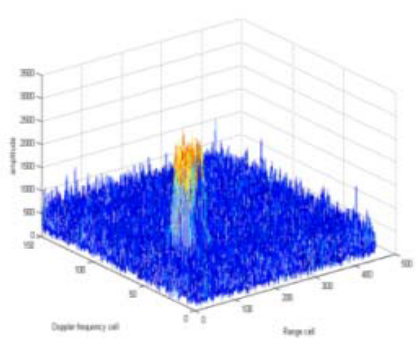

(c) 
Fig.5.the results of other methods: (a) The result of MTD, (b) The result of $\mathrm{KT}$, (c) The result of method in [10]

\section{CONCLUSION}

This paper proposes a long time integration method for bistatic radar weak target detection. Without knowing the target motion information, the algorithm firstly corrects the range curvature of the target echo using the SKT. Then the quadratic phase term is estimated and compensated. After compensating the Doppler ambiguity phase term, the target energy can be integrated by FT. Simulations have shown the validity of the proposed method.

\section{References}

1. Kreucher C. Exploiting narrowband bistatic radar measurements for dismount detection and trackin $[\mathrm{J}]$.IEEE antennas and propagation magazine, 2011, 53( 1) : 98-105.

2. Hussain M. Ambiguity functions for monostatic and bistatic radar systems using UWB throb signal $[\mathrm{J}]$. IEEE trans on aerospace and electronic systems. 2011, 47 (3) : 1710-1722.

3. Christiansen J, Olsen K. Range and Doppler walk in DVB-T based passive bistatic radar [C] . IEEE international conference, 2010, 620-626.

4. Skolnik M. Role of radar in microwaves $[\mathrm{J}]$. IEEE trans on microwave theory and techniques, 2002, 50 ( 3 ) :625-632.

5. Carretero-Moya J , Gismero-Menoyo J , Asensio-Lopez A, et al. Small-target detection in high-resolution heterogeneous sea-clutter: an empirical analysis $[\mathrm{J}]$. IEEE transactions on aerospace and electronic systems, 2011 , 47( 3) :1880-1898.

6. Carlson B D, Evans E D, Wilson S L. Search radar detection and track with the Hough transform, Part I: system concept $[\mathrm{J}]$. IEEE transactions on aerospace and electronic systems. 1994, 30(1):102-108.

7. Mo L, Wu S, Li H. Radar detection of range migrated weak target through long-term integration [J]. Chinese Journal of Electronics. 2003, 12(4) : 539-544.

8. R. P. Perry, R. C. Dipietro, and R. L. Fante,"SAR imaging of moving targets,"IEEE Transactions on
Aeropace and Electronic Systems, vol.35, no.1, pp.188-200, January 1999.

9. J. Xu, J. Yu, Y. N. Peng, and X. G. $\mathrm{Xia}$,"Radon-Fourier transform(RFT) for radar target detection(I): Generalized Doppler filter bank processing,"IEEE Transaction on Aerospace and Electronic Systems, Vol. 47, no. 2, pp. 1186-1202, April 2011.

10. D.Kirkland,"Imaging moving targets using the second-order keystone transform," IET Radar Sonar and Navigation, vol. 5, no. 8, pp. 902-910, October 2011.

11. F. Comblet, A. Khenchaf, A. Baussard, and F. Pellen, "Bistatic synthetic aperture radar imaging: Theory, simulations and validations," IEEE Trans.Antennas Propag., vol. 54, no. 11, pp. 3529-3540, Nov. 2006.

12. Li Ya-chao, Su Jun-hai, and Xing Meng-dao, et al.. Research on ISAR imaging of the complexly moving target based on the time-chirp distribution [J]. Journal of Xidian University, 2008, 35(1): 1-7. 